\title{
Evaluation of Neck Lymph Node Metastasis on Contrast-Enhanced Ultrasound: An Animal Study
}

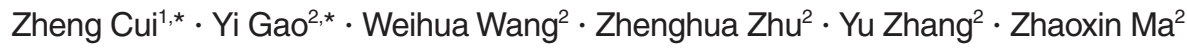 \\ Departments of ${ }^{1}$ Ultrasound Diagnosis and ${ }^{2}$ Otorhinolaryngology-Head and Neck Surgery, East Hospital, Tongji University School of Medicine,
}

Shanghai, China

Objectives. To assess the performance of contrast-enhanced ultrasound (CEUS) intended to differentiate hyperplastic from malignant neck lymph nodes in an animal model.

Methods. Twenty-four New Zealand rabbits were randomly divided into two groups: neck lymph node metastasis group (12 rabbits) and reactive hyperplastic lymph node group (12 rabbits). Tongue VX2 carcinoma with cervical lymph node metastasis was induced in 12 rabbits by injecting VX2 carcinoma suspension into the left tongue submucosa. Hyperplastic neck lymph nodes were induced by injecting egg yolk in the submandibular region of the rabbits in hyperplastic group. CEUS were performed in both groups before and after intravenous administration of SonoVue. The site, number, echogenicity, longitudinal and transverse nodal dimensions, patterns of enhancement of the neck lymph nodes were observed and recorded.

Results. In both groups only one lymph node was found in the left (tumor) side of the neck. CEUS found 12 of 12 metastatic lymph nodes in metastasis group, and diagnosed 11 of 12 lymph nodes as metastatic. Histopathologic analysis revealed metastatic lesions in all 12 rabbits, each with one lymph node, and all 12 lymph nodes in hyperplastic group is inflammation lymph nodes. All 12 cases in the hyperplastic group showed centripetal homogeneous enhancement while in the metastasis group one case showed centripetal homogeneous enhancement, three cases showed centrifugal heterogeneous enhancement, and eight cases showed diffused heterogeneous enhancement. Only one lymph node was dissected on the left side of the neck in each rabbit in both groups.

Conclusion. CEUS can play a role in discriminating metastatic from hyperplastic lymph nodes in head and neck carcinoma.

Keywords. Ultrasonography; Head and Neck Neoplasms; Lymph Node Metastasis

- Received August 23, 2015

Revised November 3, 2015

Accepted March 10, 2016

- Corresponding author: Yu Zhang

Department of Otorhinolaryngology-Head and Neck Surgery, East Hospital,

Tongji University School of Medicine, 150 Jimo Road, Shanghai 200120,

China

Tel: +86-15001854851, Fax: +86-21-58798999

E-mail: faust009@163.com

- Co-corresponding author: Zhaoxin Ma

Department of Otorhinolaryngology-Head and Neck Surgery, East Hospital, Tongji University School of Medicine, 150 Jimo Road, Shanghai 200120, China

Tel: +86-13310110889, Fax: +86-21-58798999

E-mail: mzhx114@163.com

*The first two authors contributed equally to this study.

\section{INTRODUCTION}

Although diagnosing metastasis of an enlarged lymph node with noninvasive investigations such as computed tomography (CT) or magnetic resonance imaging (MRI) is relatively easy, determining malignancy in occult neck metastasis is difficult [1]. Conventional gray-scale sonography have been widely used in evaluating superficial lymph nodes [2,3]. Ultrasonography is superior to $\mathrm{CT}$ and MRI in the aspect of evaluating internal structures of lymph nodes [4]. The absence of the echogenic hilus, round shape (longitudinal diameter-to-transverse diameter ratio of $<2$ ), and peripheral capsular vascularization are characteristic signs of metastatic lymph nodes [5]. However it is also unable to

Copyright $\odot 2017$ by Korean Society of Otorhinolaryngology-Head and Neck Surgery.

This is an open-access article distributed under the terms of the Creative Commons Attribution Non-Commercial License (http://creativecommons.org/licenses/by-nc/4.0)

which permits unrestricted non-commercial use, distribution, and reproduction in any medium, provided the original work is properly cited. 
depict small neoplastic deposits in metastatic lymph nodes with no morphological change. By using a microbubble contrast agent, contrast-enhanced ultrasound (CEUS) allows enhanced signal intensity from micro vessels, therefore better visualization of vascularization [6]. Compared with conventional ultrasound, CEUS has been reported to be able to improve the results of evaluating enlarged lymph nodes [7].

The aim of this study was to assess the ability of CEUS in noninvasively differentiating hyperplastic from malignant cervical lymph nodes with no morphological change. In this study, metastatic cervical lymph node models were established in New Zealand white rabbits by primary tumor induction in tongue. VX2 carcinoma exhibit early lymphatic metastasis, which is similar in human head and neck squamous cell carcinomas, making it a suitable model [8].

\section{MATERIALS AND METHODS}

\section{Establishing animal model}

This study was conducted under the approval of the Animal Care and Use Committee of Tongii University. Twenty-four New Zealand white rabbits weighted 2.5 to $3.0 \mathrm{~kg}$ were randomly divided into two groups: a tongue carcinoma with cervical lymph node metastasis group (metastasis group, 12 rabbits) and a reactive hyperplastic neck lymph node group (hyperplastic group, 12 rabbits). In metastasis group, tongue carcinoma and cervical lymph node metastasis were induced by VX2 mass suspension injection, the tongue was pulled out and approximately $0.2 \mathrm{~mL}$ of VX2 carcinoma suspension was injected into the left tongue ventrolateral submucosa [9]. To induce reactive hyperplastic lymph node in the hyperplastic group, $0.5 \mathrm{~mL}$ of egg-yolk emulsion was injected subcutaneously into the left submandibular region. All transplants were performed under general anesthesia with the rabbits restrained in the supine position on a specially designed operating table.

\section{Sonographic contrast agent}

The sonographic contrast agent SonoVue (Bracco, Milan, Italy) used in this study was a microbubble suspension. Constituted by stabilized microbubbles, SonoVue is provided as a lyophilized powder in a septum-sealed vial, which presents stability and re-

\section{H I $G$ G H L I I G}

- Contrast-enhanced ultrasound (CEUS) allows better identification of the vascular anatomy and lymph node perfusion.

- Hyperplastic and malignant lymph nodes show different enhancement patterns.

- CEUS have the ability of evaluating cervical lymph nodes with no morphological change. sistance to pressure. The agent was reconstituted by injecting 5.0 $\mathrm{mL}$ of saline into the septum, followed by gentle hand shaking of the vial. The bubble concentration was in the range of (1-5) $10^{8}$ microbubbles $/ \mathrm{mL}$ and the bubble size extends from approximately 0.7 to $10 \mu \mathrm{m}$ [10]. In this study, the contrast agent was administered intravenously in the right auricular vein of the New Zealand rabbit.

\section{Sonographic equipment}

Both conventional and CEUS tests were performed with a Philips IU-22 scanner (Philips Healthcare, Bothell, WA, USA). A broadband linear-array transducer (L9-12 MHz for conventional ultrasound and CEUS) was used in this study. The scanning settings of the scanner, including the gain, scanning depth, focus and time gain control were optimized for each region independently and remained unchanged throughout the study of that region. The focus was set at the deeper aspect of the lesion being examined. CEUS was carried out using contrast tuned imaging mode with low acoustic power (a mechanical index of 0.1).

\section{Gray-scale CEUS}

Before the ultrasound examinations, the animals were anesthetized with an intramuscular injection of $30 \mathrm{mg}$ of pentobarbital sodium and $5 \mathrm{mg}$ of diazepam per kilogram of body weight.

The rabbit was fixed to a specially designed device in a supine position with the neck extended and the hair of front neck region was cleared with $8 \%$ sodium sulfide solution to minimize the difference in acoustic impedance between the transducer and the skin.

Before injection of SonoVue (Bracco), conventional ultrasonography was performed in an attempt to identify the lymph nodes in the left side (tumor side) of neck region. Then $0.6 \mathrm{~mL}$ of SonoVue bolus was administered intravenously in the right auricular vein. Then gray-scale CEUS was performed in the left frontal neck region in an attempt to identify the lymph nodes. The site, number, echogenicity, margin sharpness, longitudinal and transverse nodal dimensions, contrast-enhanced patterns of the lymph nodes were observed and recorded, and a mark was made on the skin immediately above the enhanced nodes. Following characters of enhancement patterns of the lymph nodes were obtained by direct visualization: enhancement order (centrifugal, centripetal, and diffuse) and contrast distribution patterns (homogeneous, heterogeneous, or scarce). The arrival time (AT), time to peak intensity (PT), and peak signal intensity (PI) were recorded. PI was obtained by a semi-quantitative method (set peak signal intensity of nearby vessel after injection of SonoVue [Bracco], as high intensity).

Evaluated by visualization of contrast distribution patterns, hyperplastic and metastatic lymph nodes were differentiated. The lymph nodes that showed centripetal, heterogeneous, or scarce perfusion were defined as metastatic lymph nodes, and the lymph nodes with centrifugal perfusion, homogeneous or 

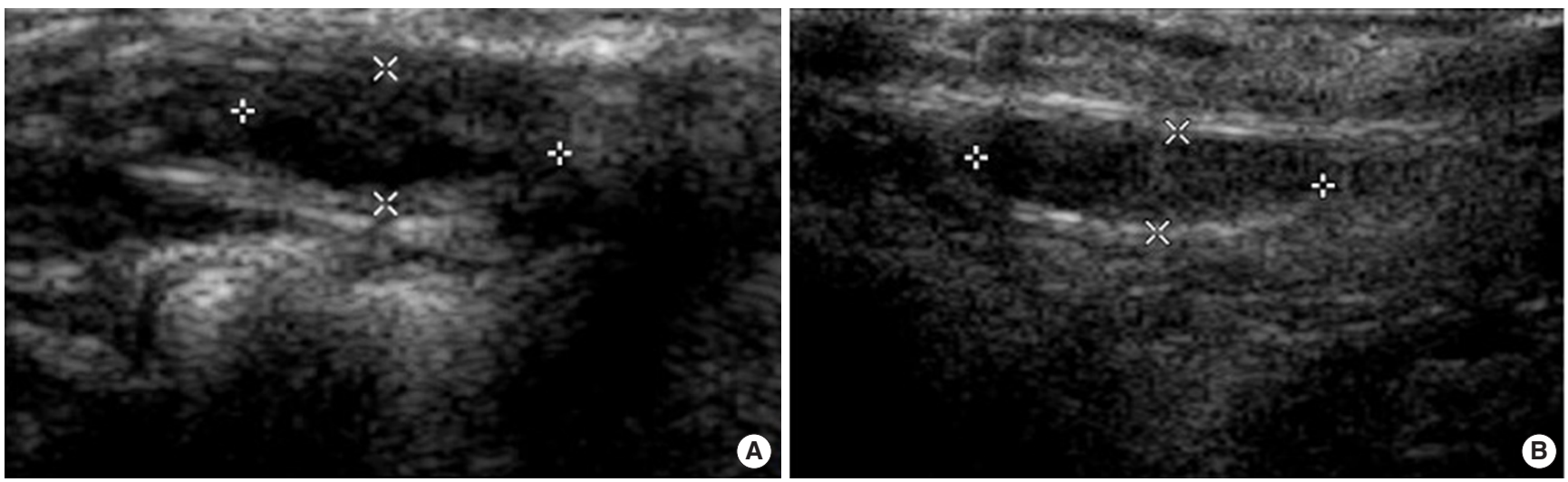

Fig. 1. Conventional ultrasonography before injection of Sonovue (Bracco, Milan, Italy). (A) Hyperplastic group and (B) metastasis group. Both group showed an oval shaped smooth bordered low echogenicity, but whether the lymph nodes were hyperplastic or malignant cannot be evaluated.
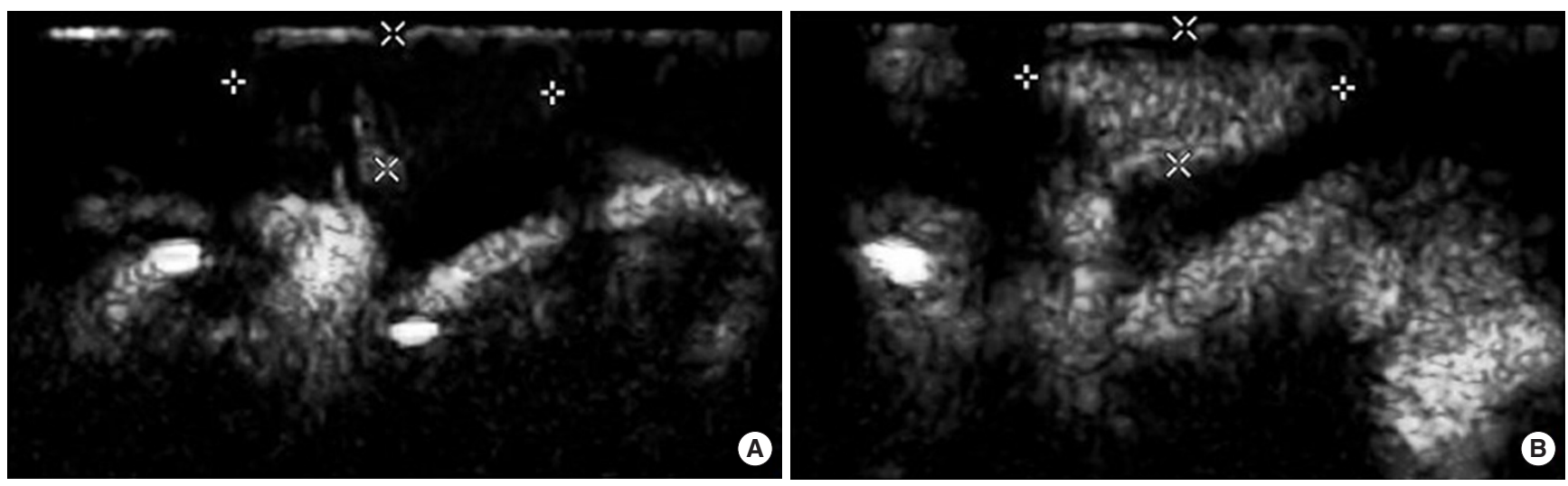

Fig. 2. The ultrasonic appearance of a lymph node in the hyperplastic group after the injection of Sonovue (Bracco, Milan, Italy). Contrast enhanced sonogram showed intense and homogenous enhancement of lymph node echogenicity, and the enhancement starts from the central area of the lymph node (centrifugal enhancement). (A) Five seconds after the injection and (B) 10 seconds after the injection.

centrifugal enhancement patterns were recognized as hyperplastic lymph nodes.

\section{Intraoperative lymph nodes identification}

Animals were sacrificed by overdose of anesthesia after CEUS. An incision was made at the marked site and the lymph nodes were identified and resected with the examiner blind to the results of CEUS. After the removal of lymph nodes, complete lymph node dissection of the left frontal neck region was performed.

\section{Statistical analysis}

SPSS ver. 18.0 (SPSS Inc., Chicago, IL, USA) was used for the data analysis. Data were analyzed by a Fisher exact test to detect if the enhancement patterns are different after CEUS between hyperplastic group and metastatic group. A significance level of $\mathrm{a}=0.05$ was used. We compared the AT and PT value between the two groups using a Student $t$-test $(P<0.05)$.

\section{RESULTS}

\section{Identification of lymph nodes with CEUS}

Unlike human lymph nodes, the ratio of longitudinal diameter to transverse diameter of normal rabbit deep cervical lymph nodes is about $4: 1$, and the ratio is similar in metastatic ones. In our study, conventional ultrasonography identified one lymph node lateral to the larynx-trachea region in the left side (tumor side) of the neck in both the metastasis group and the hyperplastic group, with an oval shaped smooth bordered low echogenicity (Fig. 1). The longitudinal diameter-to-transverse diameter ratio is about 4:1, and no vascular structures can be detected. But whether the lymph nodes were hyperplastic or malignant cannot be evaluated.

After the intravenous administration of SonoVue, CEUS revealed 12 lymph nodes in both the metastatic group and hyperplastic group, each with one oval shaped lymph node in the left side of the neck (tumor side). In hyperplastic group, all lymph nodes were enhanced homogeneously, no filling defect was vi- 

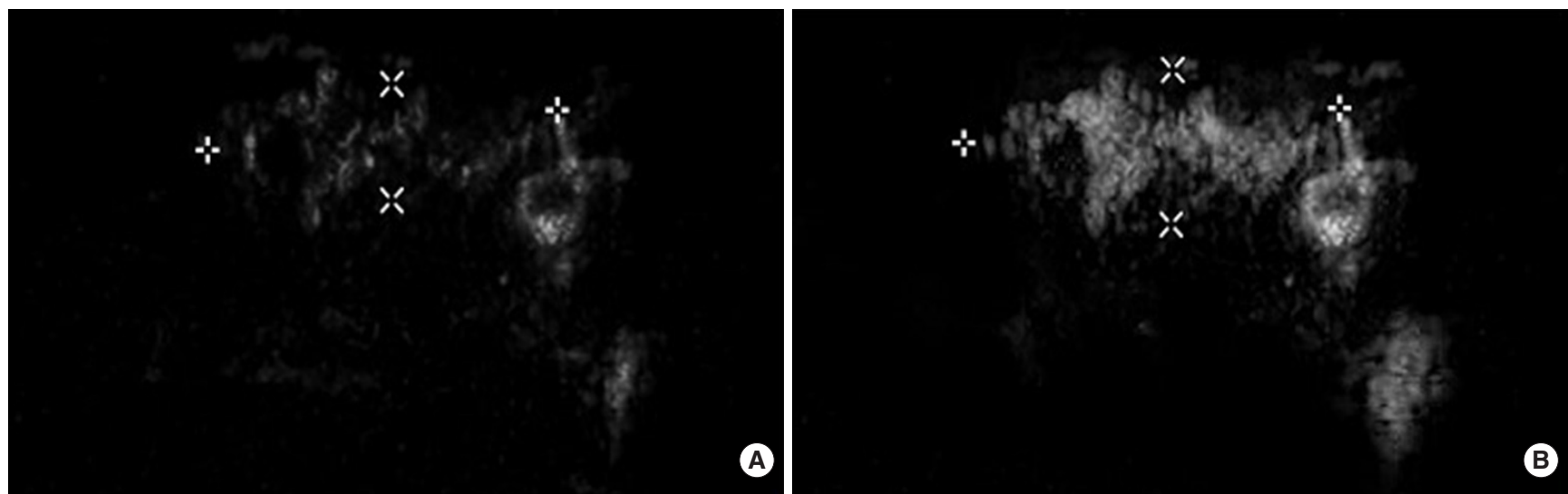

Fig. 3. The ultrasonic appearance of a lymph node in the metastasis group after the injection of Sonovue (Bracco, Milan, Italy). Contrast enhanced sonogram showed heterogeneous enhancement of lymph node echogenicity, and the enhancement started from both the central and peripheral area of the lymph node (diffuse enhancement). (A) Three seconds after the injection and (B) 10 seconds after the injection.
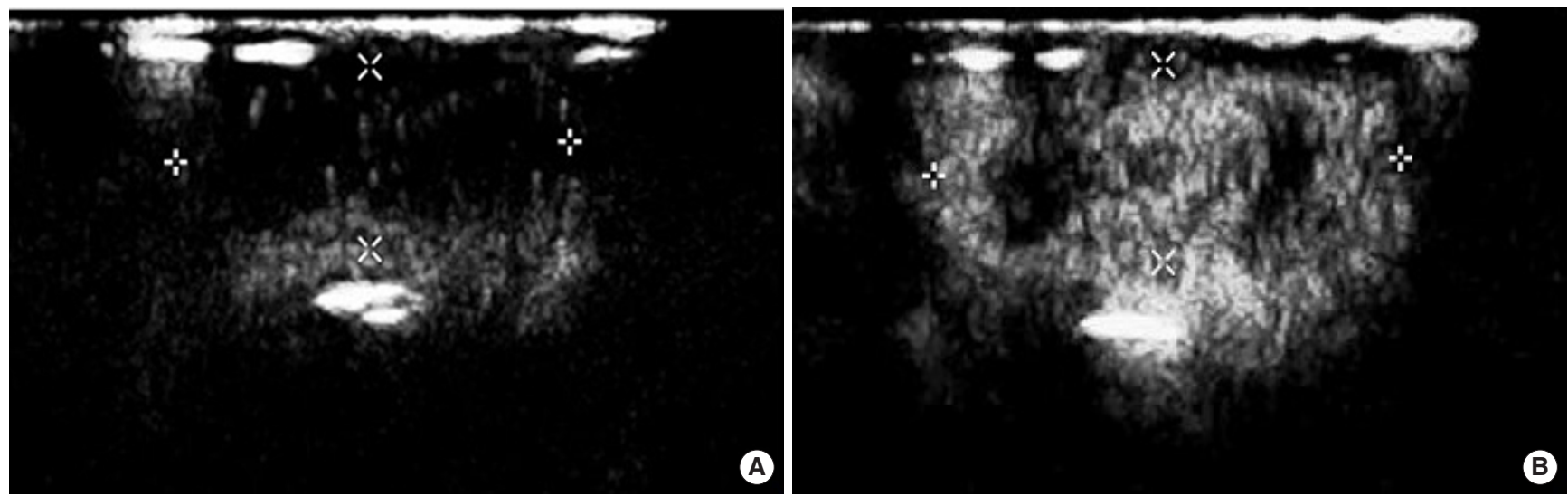

Fig. 4. The ultrasonic appearance of another lymph node in the metastasis group after the injection of Sonovue (Bracco, Milan, Italy). Contrast enhanced sonogram showed heterogeneous enhancement of lymph node echogenicity, and the enhancement started from the peripheral area of the lymph node (centripetal enhancement). (A) Three seconds after the injection and (B) 10 seconds after the injection.

Table 1. Enhancement patterns in two groups

\begin{tabular}{|c|c|c|c|c|c|c|c|c|}
\hline \multirow{2}{*}{ Group } & \multirow{2}{*}{ No. } & \multicolumn{2}{|c|}{ Enhancement type } & \multicolumn{3}{|c|}{ Enhancement order } & \multicolumn{2}{|c|}{ Enhancement degree } \\
\hline & & Homogenous & Heterogeneous & Centrifugal & Centripetal & Diffuse & Low-medium & Medium-high \\
\hline Hyperplastic & 12 & 12 & 0 & 12 & 0 & 0 & 0 & 12 \\
\hline Metastatic & 12 & 1 & 11 & 1 & 3 & 8 & 9 & 3 \\
\hline
\end{tabular}

Enhancement types, contrast distribution patterns and enhancement degrees are significantly different (Fisher exact test, $P<0.05$ ).

sualized, and enhancement patterns were characterized as centrifugal (Fig. 2). While in the metastasis group one case showed homogeneous centrifugal enhancement, of the 11 cases showed inhomogeneous enhancement (Fig. 3), three cases were centrifugal, and eight were diffuse (Fig. 4). The enhancement patterns between the two groups are significantly different (Fisher exact test) (Table 1). Hyperplastic lymph nodes are characterized by homogeneous centrifugal vessel anatomy, while metastases ones shows a heterogeneous centripetal enhancement. The AT, PT, and PI were not significantly different (Table 2).
Table 2. Enhancement time in two groups

\begin{tabular}{lccc}
\hline Group & No. & AT $(\mathrm{sec})$ & PT $(\mathrm{sec})$ \\
\hline Hyperplastic & 12 & $6.75 \pm 1.67$ & $12.38 \pm 1.50$ \\
Metastatic & 12 & $7 \pm 2.22$ & $18.75 \pm 5.93$ \\
$t$-value & & 0.255 & 0.907 \\
$P$-value & & $>0.5$ & $>0.2$ \\
\hline
\end{tabular}

No significant difference between the two groups in AT and PT value ( $t$ test, $P>0.05$ ).

AT, arrival time; PT, time to peak intensity. 


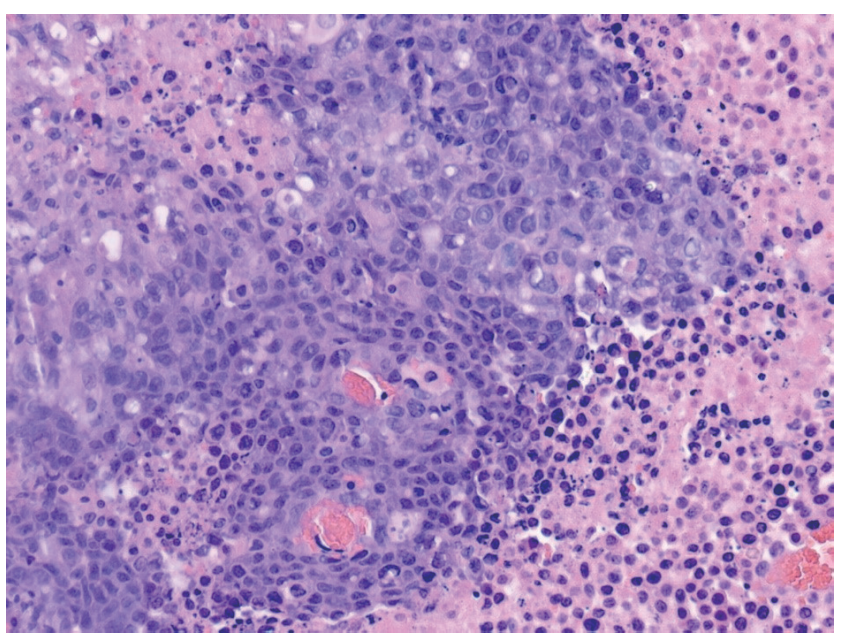

Fig. 5. Lymph node metastasis of poorly differentiated squamous cell carcinoma with partial necrosis $(H \& E, \times 200)$.

\section{Perioperative lymph node identification}

An incision was made at the site marked during CEUS and only one lymph node was identified in left side of the neck, with an identification rate of $100 \%$. The location of the lymph nodes was consistent with that of the enhanced lymph nodes on the CEUS images. The average short diameter of the lymph nodes were $0.79 \pm 0.07 \mathrm{~cm}$ and $0.78 \pm 0.09 \mathrm{~cm}$, in hyperplasic and metastasis group, respectively.

\section{Pathological results}

The presence of tongue carcinoma induced by injection of tumor suspension in the metastasis group was verified by pathological examination. All resected nodes were confirmed to be lymph nodes histologically (Fig. 5). Histological analysis revealed metastases in the left deep cervical lymph nodes in all rabbits in metastasis group, with a metastasis rate of $100 \%$.

\section{DISCUSSION}

Oral squamous cell carcinoma is associated with a high incidence of cervical lymph node metastasis, the occult metastasis rate is up to $50 \%$ in patients with $\mathrm{cN0}$ tongue cancer [11]. The presence of cervical metastasis is the most important prognostic factor in head and neck cancer patients, reducing the survival to $50 \%$ compared with patients without nodal involvement (N0).

Noninvasive investigations such as clinical examination, CT, and MR are useful methods in diagnosing enlarged metastatic cervical lymph nodes [12]; however, depictions of occult metastases in cN0 lymph nodes remains nearly impossible [1].

Conventional sonography can accurately evaluate enlarged superficial lymph nodes, but it is also unable to depict metastases without actual morphological alteration of the lymph nodes [5]. In an attempt to overcome this limitation, CEUS has been used for the study of superficial lymph nodes. By enhancing the signal intensity from micro vessels, CEUS allows better identification of the vascular anatomy [6].

Contrast enhancement patterns can provide valuable diagnostic information for differential diagnosis of hyperplastic and malignant lymph nodes. Due to intense vascularization with a rich cortical capillary circulation, reactive lymph nodes show diffused intense and homogeneous enhancement [13]. Metastatic lymph nodes are generally less vascularized than reactive lymph nodes; therefore, they show lower enhancement intensity or even perfusion deficits after contrast agent injection. Furthermore, large areas of metastasis infiltration can be caused by scarce or absent of perfusion, which is not uncommon in metastatic lymph nodes [14].

Rubaltelli et al. [7] examined 56 lymph nodes from 45 patients with CEUS, and found that 28 of 30 reactive lymph nodes showed intense homogeneous enhancement, 17 lymph nodes showed perfusion defects, of which 15 were neoplastic and two were inflammatory; four metastatic lymph nodes showed scarce or absent intranodal enhancement, and consider the technique to be able to afford a higher degree of accuracy than conventional ultrasonography in the differential diagnosis between hyperplastic and metastatic superficial lymph nodes by assessment of characteristic enhancement patterns. In a study of 94 superficial enlarged lymph nodes in 94 patients, Yu et al. [15] indicate that CEUS has a higher degree of diagnostic accuracy than conventional ultrasonography for evaluations of superficial lymphadenopathy. CEUS have been used in evaluation of the morphology and structure of lymph nodes.

However, there are few studies on evaluation of neck hyperplastic and metastatic lymph nodes without morphological change via CEUS. In our study, we found that the enhancement patterns were significantly different between hyperplastic and metastatic lymph nodes. Hyperplastic lymph nodes are characterized by homogeneous centrifugal vessel anatomy, while metastases ones shows a heterogeneous centripetal enhancement pattern. One case in metastasis group showed diffused centrifugal enhancement, which we considered to be false-negative for metastasis. This may be due to the fact that metastasis is too small to alter the vascularization, therefore not to be able to be depicted by CEUS. Therefore the size of metastatic lesion that can be depicted by CEUS still requires further study.

The study showed no significant difference in AT and PT between hyperplastic group and metastatic group, which was similar to other studies $[16,17]$. The PT reflected the perfusion rate of microbubbles. However, the specific interpretation is still unclear.

Our study does have some limitations. First, the perfusion characteristics of the lymph nodes examined were correlated with the histologic diagnoses, but no correlation was made between virtual lymph node and histologic section to match areas of metastasis and necrosis. Second, our study has only used a numerically limited series of cases, in order to find more perfusion patterns, further studies that include a large number of cas- 
es is necessary. Third, CEUS does have some intrinsic limitations remain to be considered. The results obtainable with this technique depend on the subjective evaluation of the sonographer. And US lack the ability to get a panoramic view of both the primary tumor and the metastatic lesion. Besides, it is impossible to evaluate deep-seated lymph node groups in human.

In conclusion, the experiment demonstrated the ability of CEUS to evaluate lymph node perfusion, thereby obtaining a higher level of diagnostic accuracy in differentiating hyperplastic form metastatic lymph nodes without morphological change. Furthermore, the application of CEUS in guiding fine needle aspiration may also provide a more efficient and safe method in the diagnosis of lymph node metastasis than conventional ultrasound guided fine needle aspiration.

Additional research is needed to find more patterns of CEUS enhancement, and to determine if this new method of metastasis lymph node evaluation can be used in clinical applications.

\section{CONFLICT OF INTEREST}

No potential conflict of interest relevant to this article was reported.

\section{ACKNOWLEDGMENTS}

The project supported by Key Disciplines Group Construction Project of Pudong Health Bureau of Shanghai (PWZxq2014-09), Shanghai, China. The funders had no role in study design, data collection and analysis, decision to publish, or preparation of the manuscript.

\section{REFERENCES}

1. Shoaib T, Soutar DS, MacDonald DG, Camilleri IG, Dunaway DJ, Gray HW, et al. The accuracy of head and neck carcinoma sentinel lymph node biopsy in the clinically N0 neck. Cancer. 2001 Jun;91 (11):2077-83.

2.Ahuja A, Ying M.An overview of neck node sonography. Invest Radiol. 2002 Jun;37(6):333-42.
3. Ahuja A, Ying M. Sonography of neck lymph nodes. Part II: abnormal lymph nodes. Clin Radiol. 2003 May;58(5):359-66.

4. Sumi M, Ohki M, Nakamura T. Comparison of sonography and CT for differentiating benign from malignant cervical lymph nodes in patients with squamous cell carcinoma of the head and neck. AJR Am J Roentgenol. 2001 Apr;176(4):1019-24.

5. Rubaltelli L, Corradin S, Dorigo A, Tregnaghi A, Adami F, Rossi CR, et al. Automated quantitative evaluation of lymph node perfusion on contrast-enhanced sonography. AJR Am J Roentgenol. 2007 Apr; 188(4):977-83.

6. Santamaria G, Velasco M, Farre X, Vanrell JA, Cardesa A, Fernandez PL. Power Doppler sonography of invasive breast carcinoma: does tumor vascularization contribute to prediction of axillary status? Radiology. 2005 Feb;234(2):374-80.

7. Rubaltelli L, Khadivi Y, Tregnaghi A, Stramare R, Ferro F, Borsato S, et al. Evaluation of lymph node perfusion using continuous mode harmonic ultrasonography with a second-generation contrast agent. J Ultrasound Med. 2004 Jun;23(6):829-36.

8. Dunne AA, Mandic R, Ramaswamy A, Plehn S, Schulz S, Lippert $\mathrm{BM}$, et al. Lymphogenic metastatic spread of auricular VX2 carcinoma in New Zealand white rabbits. Anticancer Res. 2002 NovDec;22(6A):3273-9.

9. Ying HY, Wu HT, Zhou L. Establishment of the deep cervical lymph node metastasis model of tongue VX2 carcinoma and observation of its metastatic features. Zhonghua Er Bi Yan Hou Tou Jing Wai Ke Za Zhi. 2008 Oct;43(10):778-81.

10. Gorce JM, Arditi M, Schneider M. Influence of bubble size distribution on the echogenicity of ultrasound contrast agents: a study of SonoVue. Invest Radiol. 2000 Nov;35(11):661-71.

11. Yoshida K, Kashima K, Suenaga S, Nomi N, Shuto J, Suzuki M. Immunohistochemical detection of cervical lymph node micrometastases from T2N0 tongue cancer. Acta Otolaryngol. 2005 Jun;125(6): 654-8.

12. Kau RJ, Alexiou C, Stimmer H, ArnoldW. Diagnostic procedures for detection of lymph node metastases in cancer of the larynx. ORL J Otorhinolaryngol Relat Spec. 2000 Jul-Aug;62(4):199-203.

13. Gadre A, Briner W, O’Leary M. A scanning electron microscope study of the human cervical lymph node. Acta Otolaryngol. 1994 Jan;114(1):87-90.

14. Steinkamp HJ, Wissgott C, Rademaker J, Felix R. Current status of power Doppler and color Doppler sonography in the differential diagnosis of lymph node lesions. Eur Radiol. 2002 Jul;12(7):1785-93.

15. Yu M, Liu Q, Song HP, Han ZH, Su HL, He GB, et al. Clinical application of contrast-enhanced ultrasonography in diagnosis of superficial lymphadenopathy. J Ultrasound Med. 2010 May;29(5):735-40.

16. Ouyang Q, Chen L, Zhao H, Xu R, Lin Q. Detecting metastasis of lymph nodes and predicting aggressiveness in patients with breast carcinomas. J Ultrasound Med. 2010 Mar;29(3):343-52.

17. Yang WT, Metreweli C, Lam PK, Chang J. Benign and malignant breast masses and axillary nodes: evaluation with echo-enhanced color power Doppler US. Radiology. 2001 Sep;220(3):795-802. 